\title{
Multiplicity and theremalization time in heavy-ions collisions
}

\author{
Irina Aref'eva ${ }^{1, \star}$ \\ ${ }^{1}$ Steklov Mathematical Institute, Russian Academy of Sciences, \\ Gubkina str. 8, 119991, Moscow, Russia
}

\begin{abstract}
We present a concise review of quark-gluon plasma formation in heavy-ions collisions within the holographic approach. In particular, we discuss how to get the total multiplicity in heavy ions collision to fit the recent experimental data. We also discuss theoretical estimations of time formation of QGP in heavy ions collision and show that different observables can give the different times of QGP formation.
\end{abstract}

\section{Introduction}

It is my pleasure to give a talk here. I would like to thanks the organizers for invitation.

The subject of my talk is holography description of quark-gluon plasma (QGP) formation in heavy-ions collisions (HIC). The plan of the talk is the following.

- I'll start from the physical picture of formation of QGP in HIC.

- Then I'll remind the raisons to use dual approach to physical problems related with QGP. As to holographic approach to QGP there are two options:

- so called the top-down approach,

- as well as the bottom-up approach.

The meaning is obvious from the names. The top theory is of course, the string theory :). There is considerable progress in applications of holography to static properties of QGP [1]

- The main part of the talk will be devoted to discussion of the holographic description of the process of formation of QGP. The goal will be to fit experimental date related with multiplicity and thermalization time in heavy ions collisions. By experimental data I mean data from RHIC and more recent data from LHC. One can find the holographic description of QGP formation in $[2,3]$.

\subsection{Physical picture of QGP formation in heavy ions collisions}

Let me start from the physical picture of QGP formation in heavy ions collisions.

In QCD we have quark confinement and asymptotic freedom. It is known (mainly due to lattice calculations) that if the temperature $\mathrm{T}$ increases, or density nuclear matter increases, then the nuclear matter undergoes to a phase transition to the deconfined phase. The quark gluon plasma is a macroscopic matter formed from the quarks and gluons at high temperature.

^e-mail: arefeva@mi.ras.ru 

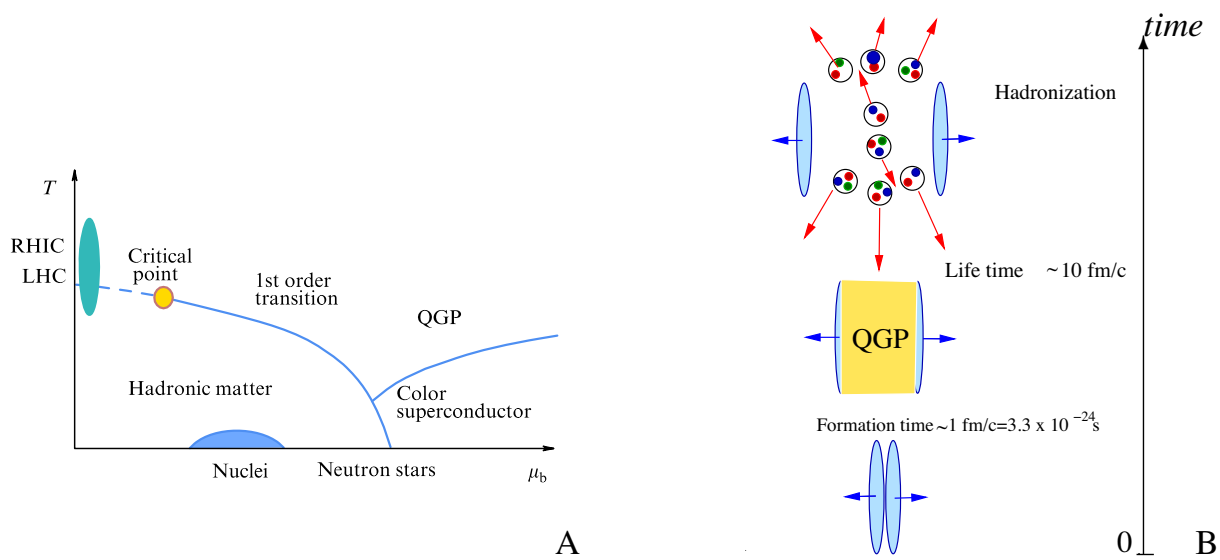

Figure 1. (color on line) A. The QCD phase diagram. B. The schematic picture of heavy ions collision

The phase diagram of QCD is not well known either experimentally or theoretically. A commonly conjectured form of the phase diagram, temperature $\mathrm{T}$ vs quark chemical potential $\mu$, is shown in Fig.1.A. The chemical potential $\mu$ is a measure of the imbalance between quarks and antiquarks in the system. The phase transition is not sharp and it is supposed to be the 1-st order.

Ordinary nuclear matter in this diagram is at $\mu=310 \mathrm{MeV}$ and $\mathrm{T}$ close to zero. If we increase the quark density, i.e. increase $\mu$, keeping the temperature low, we go into a phase of more and more compressed nuclear matter neutron stars. Above the (blue on the on-line version of the paper) smeared line there is a transition to the quark-gluon plasma. At ultra-high densities one expects to find the phase of color-superconducting quark matter. In ultra-relativistic heavy ion collisions one studies this matter in the regime of extreme energy density. In the figure the typical values of $\mu$ and $T$ in heavy-ion collisions are shown by a filled (blue) region near the T-axis.

Heavy-ions collisions are studied in several experiments. Below I list them with indication of the energy per nucleon.

- At the Brookhaven Alternating Gradient Synchrotron (AGS), started in the 1990's, the the energy per one nucleon is $\sqrt{s_{N N}}=4.75 \mathrm{GeV}$,

- At the CERN Super Proton Synchrotron (SPS), $\sqrt{s_{N N}}=17.2 \mathrm{GeV}$.

- At the Brookhaven Relativistic Heavy-Ion Collider (RHIC), $\sqrt{s_{N N}}=200 \mathrm{GeV}$

- At the LHC collider at CERN, $\sqrt{s_{N N}}=2.76 \mathrm{TeV}$ (2011), $\sqrt{s_{N N}}=5.02 \mathrm{TeV}$ (2015).

At RHIC the ions of Au are collided and at LHC the ions of $\mathrm{Pb}$ are collided. Schematically the picture of heavy ion collisions is presented in Fig.1.B.

There are strong experimental evidences that RHIC or LHC have created some medium which behaves collectively. They are the following

- modification of particle spectra (compared to $\mathrm{p}+\mathrm{p}$ ) 
- high $p_{T}$-suppression of hadrons

- jet quenching

- elliptic flow

- suppression of quarkonium production and etc.

Study of this medium is related with one of the fundamental questions in physics. This problem is what happens to matter at extreme densities and temperatures as may have existed in the first microseconds $10^{-5}, T \sim 10^{12} \mathrm{~K}$ after the Big Bang. One can say that the aim of heavy-ion physics is to collide nuclei at very high energies and create such a state of matter in the lab (Little Big Bang).

As to the theory, there are macroscopic and microscopic approaches to description of QGP formation in HIC. Macroscopic approaches include thermodynamics, hydrodynamics, kinetic theory, etc. The microscopic approach is suppose to be based on QCD, or just on some effective theories. The list of macroscopic approaches is started from the names of Fermi, Landau and Pomeranchuk, who proposed in 50-s the hydrodynamical model to high energy collisions of protons [21-23].

\subsection{Holographic approach to static QGP}

In the last more than 10 years an other approach has been developed. This approach is based on holography, and it is also known as $A d S / C F T$ approach. An application to this approach to formation of QGP in HIC is the subject of the present talk.

A remarkable conclusion from the RHIC and LHC experiments is that the QGP does not behave as a weakly coupled gas of quarks and gluons, but rather as a strongly coupled fluid. This makes perturbative methods inapplicable. The lattice formulation of QCD does not work, since we have to study real-time phenomena. This has provided a strong motivation for study the dynamics of strongly coupled quark gluon plasma through the gauge/string duality.

The Gauge/Gravity duality gives an correspondence between the 4-dim physical space where the gauge theory lives and the 5-dimensional space where the supergravity (weak curvature) approximation of the 10-dimensional string theory is valid. Or in others words, the properties of the gauge theory in (physical) Minkowski space in 3+1 dimensions are in one-to-one relation with properties of the bulk 4+1 dimensional theory.

The best known example of such theories is $\mathrm{N}=4$ super Yang-Mills, a superconformal field theory with matter in the adjoint representation of the gauge group $S U\left(N_{c}\right)$. However, there is not yet exist a gravity dual construction for QCD. Differences between $N=4$ SYM and QCD are less significant, when quarks and gluons are in the deconfined phase. Lattice calculations show that QCD exhibits a quasi-conformal behavior at temperatures $\mathrm{T}>300 \mathrm{MeV}$ and the equation of state can be approximated by e $=3 \mathrm{p}$ (a traceless conformal energy-momentum tensor), see Fig.2.A. where the comparison of the calculations carried out using the highly improved staggered quark (HISQ) and the stout-smeared staggered action (stout) for the trace anomaly, the pressure, and the entropy density are presented. These observations strongly support to use the AdS/CFT correspondence as a tool to get non-perturbative dynamics of quark gluon plasma. The AdS/CFT or holography states the relation between generation functions for correlators of QFT in the 4-dimensional Minkowski space-time and classical solutions in the 5-dimensional gravity [5], see Fig.2.B. In some sense the duality claims a kind of auto-model behaviour of strong correlator systems. There is an similarity with the auto-model behaviour of the parton model [6].

There is the considerable success in description of the static QGP. The main results are collected in the book [1]. 


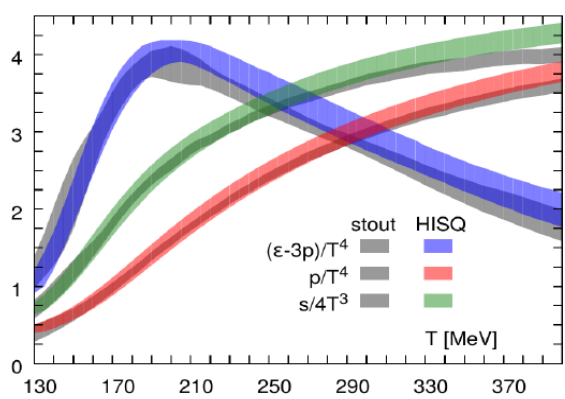

A

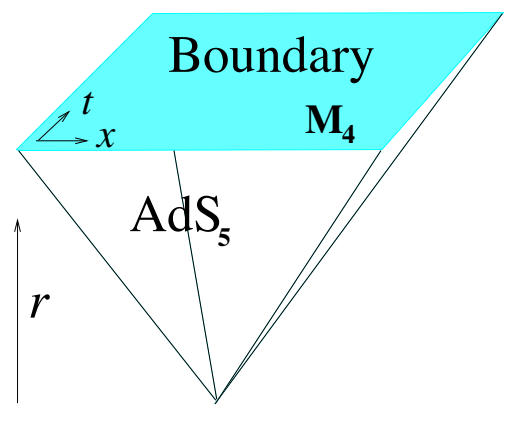

B

Figure 2. (color on line) A. The QCD equation of state from the lattice. The figure taken from Ref.[4]. B. The schematic picture for holographic calculations $(r=1 / z$ in $(1))$

\subsection{Holographic approach to QGP formation in heavy ions collisions}

I am going to discuss the formation of quark gluon plasma within AdS CFT setup. The main conjectures are the following:

- thermal d-dimensional QFT can be described by d+1 background with a black hole

- thermalization of 4-dimensional QFT, i.e. formation of 4-dimensional quark gluon plasma, corresponds to a black hole formation in $A d S_{5}$.

Let me now say few words about black holes formation in AdS. To initiate the process of BH formation one has to perturb the initial AdS metric. According to AdS/CFT correspondence this deformation should be related with the deformation of the energy-momentum tensor of the boundary theory. So the idea is to make some perturbation of AdS metric that near the boundary mimics the heavy ions collisions and see what happens.

One can consider several deformations of AdS metric:

- a deformation by adding colliding gravitational shock waves.

- we can drop of a shell of matter with vanishing rest mass ("null dust"),

- we can study the toy 3-dim model with colliding ultra relativistic particles

It seems natural to think about an ultrarelativistic nucleus as a shock wave in 4-dimensional spacetime. One can also assume that this shock has a profile function. According AdS CFT correspondence to this energy-momentum tensor corresponds the metric of a shock wave in $\mathrm{AdS}_{5}$ of the following form

$$
d s^{2}=L^{2} \frac{-d u d v+d x_{\perp}^{2}+\phi\left(x_{\perp}, z, L\right) \delta(u) d u^{2}+d z^{2}}{z^{2}},
$$

here $u, v$ are light-cone variables, $u=x_{0}+x_{\|}, v=x_{0}-x_{\|}, \phi\left(x_{\perp}, z, L\right)$ is the profile function, $L$ is the scale parameter. For $\phi\left(x_{\perp}, z, L\right)=0$ the metric (1) is nothing but the Poincare metric for $\mathrm{AdS}_{5}$. For two colliding nuclei corresponds the following metric in $\mathrm{AdS}_{5}$

$$
d s^{2}=L^{2} \frac{-d u d v+d x_{\perp}^{2}+\phi_{1}\left(x_{\perp}, z, L\right) \delta(u) d u^{2}+\phi_{2}\left(x_{\perp}, z, L\right) \delta(v) d v^{2}+d z^{2}}{z^{2}} .
$$


It is interesting to note that gaussian regularization of the point shock wave profile function $\phi\left(x_{\perp}, 0\right)$ exactly corresponds to the Woods-Saxon profile for the nuclear density. This correspondence give us L equal to 4.3 fermi for gold and L equal to 4.4 fermi for lead.

It is natural to ask the question: can we guaranty the black hole formation under collisions of these shock waves. If the answer is "yes", then I'll take the entropy of this formed black hole as a multiplicity of particles production. This idea has been explored in several papers [7-16]. One can also study the problem numerically and a lot of efforts are performed in this direction $[17,18]$.

\section{Analytical estimation of the entropy of the $\mathrm{BH}$ produced in collision of two schock waves}

I am going to speak about what can be done analytically. As I have already mentioned in Introduction the main goal is to estimate multiplicity and thermalization time.

\subsection{Multiplicity}

The lead-lead collisions show a power form of the multiplicity dependence on the energy, and the power is about $0.15,[19,20]$

$$
\mathcal{M} \sim s^{0.15}
$$

here $s$ is the Mandelstam variable, that is equal to the square of the energy in the central mass frame, see Fig.3.
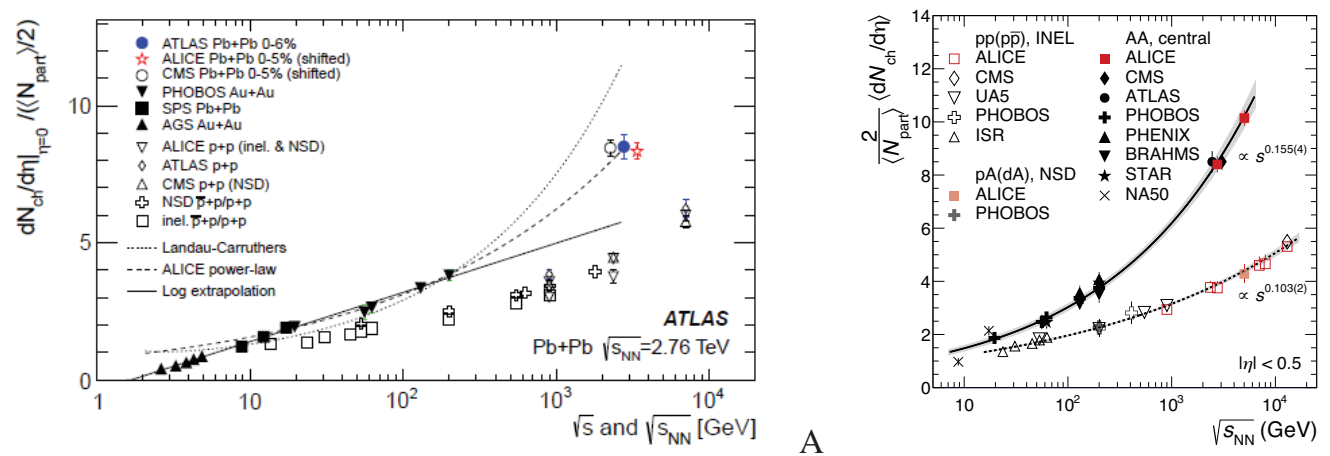

B

Figure 3. Plots of the total number of charged particles versus energy $\sqrt{s}$ for protons and $\sqrt{s_{N N}}$ per pair of nucleons for ions collisions. A. Plot from ATLAS collaboration [19]. B. Plot from ALICE collaboration [20].

The idea to relate multiplicities with the thermodynamic characteristic of the media produced as a result of a collision of particles comes back to Landau and Fermi [21-24].

The idea to estimate multiplicity by the entropy of $\mathrm{BH}$ produced in 5-dim under perturbation of the initial AdS have proposed in [7] and elaborated in [9, 11-14, 16].

The entropy is assumed to be estimated by the area of the trapped surface that forms in collision of two shock waves if the energy of colliding shocks is above a threshold. This estimation is related with the Penrose theorem that claims that black hole formation is equivalent to a trapped surface formation and the area of horizon of the formed black hole can be estimated by the trapped surface area. It worth mention that the Penrose theorem deals with asymptotically flat spaces. But we apply here this theorem to the case of the AdS space-time. This point requires more treatments. 
Now let me tell what we get in the holographic estimations of the multiplicity. The simplest holographic model gives $[7,9,13]$

$$
\mathcal{M} \sim s^{1 / 3}
$$

This is faraway from the experimental results $[19,20]$, the power is twice more. The natural idea arises to try to modify the holographic model. The simplest option is to modify the background. We can do this in several ways:

- removed parts of AdS assuming some cuts [7]

- assuming existence of some others fields and change the vacuum solution from AdS to some modification. The simplest possibility is to deal with dilaton-gravity [16, 26, 27].

The more prominent dilation-gravity model is the so called improved holographic QCD [25]. There exist the dilation potential such that the model reproduces 2-loop beta function as well as glueball spectrum. The shock waves collisions in this background have been calculated in [16]. They have improved a little the power (they also got the log corrections), but still the power is not as shown in Fig.3. In [26] it has been show that one can get the required power around $s^{0.15}$, but in this case one has to assume that the dilaton is in fact a phantom. In [27] we have shown that one can get $\mathcal{M} \sim s^{0.15}$ for $s$ bounded from above.

Since in all these considerations more or less general backgrounds reproducing AdS at UV and confinement at IR have been used, one can think that just general assumptions about the background prevent to reproduce the suitable behavior in UV and IR and in the same time give the correct energy dependence of multiplicity at high energy. In particular, we can think that a default assumption of the isotropic form of background metrics is responsible for this discrepancy and for a more realistic description of the entropy production in holographic models one has to consider anisotropic backgrounds.

\subsection{Anisotropic holographic backgrounds}

In favor of anisotropic holographic backgrounds there are also additional arguments. Up to couple years ego, it was believed that just after heavy-ion collisions, a pre-equilibrium period exists for up to $1 \mathrm{fm} / \mathrm{c}$ and then the QGP appears and this QGP is isotropic. However now there is a belief that the QGP is created after a very short time after the collision, $\tau_{\text {therm }} \sim 0.1 \mathrm{fm} / \mathrm{c}$, and it is anisotropic ("anisotropic" means to a spatially anisotropy) for a short time $\tau$ after the collision, $0<\tau_{\text {therm }}<\tau<$ $\tau_{i s o}$, and the time of locally isotropization is about $\tau_{i s o} \sim 2 \mathrm{fm} / \mathrm{c}$.

In the holographic version of this setup it is suitable to consider a black hole formation in a spatially anisotropic background. We consider one possible version of the anisotropic background, namely the so-called Lifshitz-type background

$$
d s^{2}=L^{2}\left[\frac{\left(-d t^{2}+d x^{2}\right)}{z^{2}}+\frac{\left(d y_{1}^{2}+d y_{2}^{2}\right)}{z^{2 / v}}+\frac{d z^{2}}{z^{2}}\right] .
$$

It has been shown in [30] that for the wall-on-wall collision in the 5d Lifshitz-like background with the critical exponent $v=4$, the dependence of multiplicity on the energy is desirable, i.e. behaves as $s^{1 / 6} \cdot v=1$ corresponds to the AdS case.

This metric is a solution $[30,35]$ to a $5 \mathrm{~d}$ model governed by the action

$$
S=\frac{1}{16 \pi G_{5}} \int d^{5} x \sqrt{|g|}\left(R+\Lambda-\frac{1}{6}\left(H_{(3)}^{2}+m_{0}^{2} B_{(2)}^{2}\right)\right),
$$


where $m_{0}$ and $\Lambda$ are constant and the 3 -form $H_{(3)}$ and the 2-form $B_{(2)}$ are related by $H_{(3)}=d B_{(2)}$. There is a problem with action (6) since up to now we do not know the blacking version of the metric (5) as a solution to the model (6). However there is a possibility to support the metric (5) by another action and to find the corresponding blacking solution[32]. This action has the form

$$
S=\int d^{5} x \sqrt{|g|}\left(R[g]+\Lambda-\frac{1}{2}(\partial \phi)^{2}-\frac{1}{4} e^{\lambda \phi} F_{(2)}^{2}\right),
$$

where the 2-form $F_{(2)}$ is the gauge field with $F_{(2)}^{2}=F_{m n} F^{m n}, \phi$ is the dilaton scalar field, $\lambda$ is a dilatonic coupling constant and $\Lambda$ is the cosmological constant. The model (7) can be considered as a truncated supergravity $I I A$ in the style of [35]. Another possible underlying theory is the $5 \mathrm{~d} S O(6)$ gauged supergravity [36].

The metric of the black brane solution in the theory (7) reads

$$
d s^{2}=e^{2 v r}\left(-f(r) d t^{2}+d x^{2}\right)+e^{2 r}\left(d y_{1}^{2}+d y_{2}^{2}\right)+\frac{d r^{2}}{f(r)},
$$

with the blackening function given by

$$
f(r)=1-m e^{-(2 v+2) r}
$$

and the dilaton and Maxwell fields:

$$
\phi=\phi(r), \quad e^{\lambda \phi}=\mu e^{4 r}, \quad F_{(2)}=\frac{1}{2} q d y_{1} \wedge d y_{2},
$$

where $\mu$ and $q$ are two constants. For the particular case $v=4$, the metric (8)-(9) along with the ansatz (10) solves the field equations if the constants take the following values:

$$
\lambda= \pm \frac{2}{\sqrt{3}}, \quad \Lambda=90, \quad \mu q^{2}=240 .
$$

See Appendix in [32] for details.

If the dilaton is constant and the Maxwell field vanishes, the metric (8) with $v=1$ turns out to be the black brane solution in the AdS background:

$$
d s^{2}=e^{2 r}\left(-f(r) d t^{2}+d x^{2}\right)+e^{2 r}\left(d y_{1}^{2}+d y_{2}^{2}\right)+\frac{d r^{2}}{f(r)}, \quad f(r)=1-m e^{-4 r}
$$

\subsection{The Vaidya-Lifshitz geometry}

To study the thermalization process we need to use the infalling shell approach based on the Vaidya solution. First, we introduce the coordinate $z=e^{-v r}$, which, after the rescaling $\left(t, x, y_{1}, y_{2}\right) \mapsto$ $v^{-1}\left(t, x, y_{1}, y_{2}\right)$, allows one to rewrite the metric (8) in the form

$$
d s^{2}=z^{-2}\left(-f(z) d t^{2}+d x^{2}\right)+z^{-2 / v}\left(d y_{1}^{2}+d y_{2}^{2}\right)+\frac{d z^{2}}{z^{2} f(z)}, \quad f=1-m z^{2+2 / v} .
$$

To write down the Vaidya-Lifshitz solution, one should consider the ingoing null geodesics

$$
d t+\frac{d z}{f(z)}=0
$$


and introduce the Eddington-Finkelstein coordinate system $\left(v, x, y_{1}, y_{2}, z\right)$ via

$$
d v=d t+\frac{d z}{f(z)}
$$

Owing to (15) we can represent (13) in the following form

$$
d s^{2}=-z^{-2} f(v, z) d v^{2}-2 z^{-2} d v d z+z^{-2} d x^{2}+z^{-2 / v}\left(d y_{1}^{2}+d y_{2}^{2}\right), \quad f(v, z)=1-m(v) z^{2+2 / v},
$$

where the mass function $m(v)$ determines the thickness of the shell falling along $v=0$ and captures the information about the black hole formation. For the infinite thin shell $m(v)$ has the form

$$
m(v)=M \theta(v),
$$

where $M$ is a constant and $\theta(v)$ is the Heaviside function. One can also consider a smooth function $m(v)$ and get, for instance,

$$
f(v, z)=1-\frac{m}{2}\left(1+\tanh \frac{v}{\alpha}\right) z^{2+2 / v},
$$

where $m$ and $\alpha$ are two constants.

The solution (16) interpolates between the vacuum Lifshitz-type solution (5) inside the shell $(v<$ $0)$ and the Lifshitz black brane geometry (13) outside the shell $(v>0)$.

\section{The thermalization process}

In [30] we have shown that there is a trapped surface, which forms in the collision of two shock waves in the background (5), controlled by boundary points $z_{a}$ and $z_{b}$, with $z_{a}<z_{b}$. This trapped surface defines the location of the horizon for (16).

We can consider the thermalization time $t_{\text {therm }}$ of several physical quantities, for example the twopoint correlation functions, Wilson loops or the entanglement entropy [31-33]. All these quantities we can find in the holographically within geodesics approximations. The simple case is the the thermalization of the two-point correlation function.

\subsection{Thermalization times of two-point correlators}

Calculations of the thermalization time $t_{\text {therm }}$ of two-point correlators at the scale $\ell$ is based on finding geodesics with endpoints located at the distance $\ell$ for a bulk particle. Then, the thermalization time $t_{\text {therm }}$ is the time when this geodesic covered by the shell (16).

The general case for the Lagrangian of the point-like probe has the form

$$
\mathcal{L}=\sqrt{-z^{-2} f(z) \dot{v}^{2}-2 z^{-2} \dot{v} \dot{z}+z^{-2} \dot{x}^{2}+z^{-2 / v}\left(\dot{y}_{1}^{2}+\dot{y}_{2}^{2}\right)}
$$

where $\tau$ is a parameter and $\dot{x}=\partial_{\tau} x$, etc. Here we have two possibilities for the choice of $\tau$ with respect to the transverse directions. 

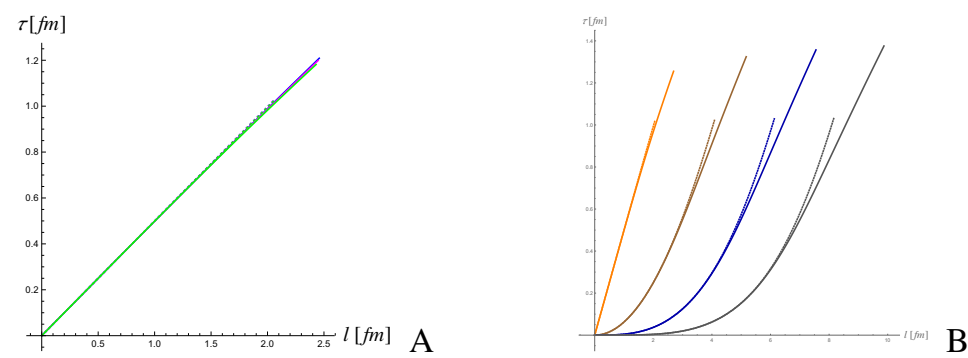

Figure 4. The thermalization time $\tau$ as a function of $\ell$ for the 5-dimensional Lifshitz metric (13)-(??) for $v=$ 2,3,4. A: Thermalization along the longitudinal direction with $m=0.5$ and $m=0.1$. All lines coincide. B: Thermalization along the transversal direction, $v=1$ (orange), $v=2$ (brown), $v=3$ (blue) and $v=4$ (gray). The solid and dotted curves correspond to $m=0.5$ and $m=0.1$, respectively.

\subsubsection{Thermalization along the longitudinal direction}

For the case $\tau=x$ ( $\mathrm{x}$ is a longitudinal direction) we have

$$
\mathcal{L}=\frac{\sqrt{\mathcal{R}_{x}}}{z}, \quad \mathcal{R}=1-f(z)\left(v_{x}^{\prime}\right)^{2}-2 v_{x}^{\prime} z_{x}^{\prime} .
$$

The integrals of motion corresponding to (20) are

$$
\mathcal{J}=-\frac{1}{z \sqrt{\mathcal{R}_{x}}}, \quad \mathcal{I}=\frac{f(z) v_{x}^{\prime}+z_{x}^{\prime}}{z \sqrt{\mathcal{R}}} .
$$

We take $\mathcal{I}=0$ and get

$$
z_{x}^{\prime}= \pm \sqrt{f(z)\left(\frac{1}{z^{2} \mathcal{J}^{2}}-1\right)} .
$$

The turning point $z_{*}$, the point there $z_{x^{*}}^{\prime}=0$, is related with $\mathcal{J}$ as $\mathcal{J}^{2}=1 / z_{*}^{2}$.

For the distance $\ell$ between the ends of the geodesic and the thermalization time one gets

$$
\begin{aligned}
\ell & =2 z_{*} \int_{0}^{1} \frac{w d w}{\sqrt{f\left(z_{*} w\right)\left(1-w^{2}\right)}}, \\
t_{\text {therm }} & =z_{*} \int_{0}^{1} \frac{d w}{f\left(z_{*} w\right)} .
\end{aligned}
$$

Note that here we assume that the turning point lies above the horizon, i.e. $z_{h}>z_{*}$. The behavior of the thermalization time as a function of the distances for (23)-(24) is represented in Fig.4.A. We see that the thermalization time behaves linearly with $\ell$. The results match to those for modified AdS models from [31] and coincide for all values of the dynamical exponent $v$.

\subsubsection{Thermalization along the transversal direction}

In this case $\tau=y_{1}$. From (19) we have

$$
\mathcal{L}=\frac{\sqrt{\mathcal{R}}}{z}, \quad \mathcal{R}=z^{2-2 / v}-f(z)\left(\dot{v}_{y}\right)^{2}-2 \dot{v}_{y} \dot{z}_{y} .
$$


The integrals of motion corresponding to (25) are

$$
\mathcal{J}=-\frac{z^{1-2 / v}}{\sqrt{\mathcal{R}}}, \quad \mathcal{I}=\frac{f(z) \dot{v}_{y}+\dot{z}_{y}}{z \sqrt{\mathcal{R}}} .
$$

For $\mathcal{I}=0$ we have the following relation between the turning point $z_{*}$ and $\mathcal{J}, \mathcal{J}^{2}=1 / z_{*}^{2 / n}$ and we get

$$
\dot{z}_{y}= \pm \frac{z^{1-\frac{2}{v}} \sqrt{f\left(1-\mathcal{J}^{2} z^{2 / v}\right)}}{\mathcal{J}} .
$$

From (27) one gets the relation between the ends of geodesic and the thermalization time

$$
\begin{aligned}
\ell & =2 z_{*}^{1 / v} \int_{0}^{1} \frac{w^{-1+2 / v} d w}{\sqrt{f\left(w z_{*}\right)\left(1-w^{2 / v}\right)}}, \\
t_{\text {therm }} & =z_{*} \int_{0}^{1} \frac{d w}{f\left(z_{*} w\right)} .
\end{aligned}
$$

Here we remove the regularization since $v>0$. The dependence (29) on (28) is given in Fig. 4.B. We see that the thermalization time in the transversal direction depends on the anizotropic parameter $v$. In particular, for $v=2$ the thermalization process is more then twice faster as compared to the longitudinal direction. By increasing $v$ we make the thermalization in the transversal direction faster. We also see that for larger values of $v$ the dependence on the mass $m$ becomes more essential.

\subsection{Thermalization time of Wilson loops}

In this section, we compare the thermalization time for spatial Wilson loops with different orientations and its dependence on the value of the dynamical exponent $v$. To simplify these estimations we consider the thin shell limit. We are interested in the value of the boundary time $t_{\text {therm }}$ when the string profile is totally covered by the thin shell, i.e.:

$$
t_{\text {therm }}(\ell)=\int_{0}^{z_{*}(\ell)} \frac{d z}{f(z)}
$$

where $\ell$ is the length between the string endpoints on the boundary.

In Fig. 5 we plot the dependence on $\ell$ of the thermalization time for two configurations in the $x y_{1}$ plane. In Fig.6.A the behavior of the thermalization time as a function of $\ell$ for the configuration in the transverse $y_{1} y_{2}$-plane is presented. One can see that the thermalization time decreases with increasing $v$ for all cases plotted in Fig. 5 and Fig. 6 . The dependence on the length $\ell$ for the loop in the $x y_{1}$-plane with the short extent in the $x$-direction is linear. At the same time, the dependence for the loop in the same plane, but with the short extent in the $y_{1}$-direction, as well as for the loop in $y_{1} y_{2}$-plane, is not linear for small $\ell$ asymptoting to the linear dependence only for large $\ell$. It should be noted that the deviation from linearity strengthens with increasing $v$. We also see that for the configuration in the transverse plane, the deviation of the thermalization time for the anisotropic cases from the isotropic one the thermalization time is much stronger than for the other orientations. In Fig.6.B the comparison of thermalization times for different orientations in the case $v=4$ is plotted. This plot shows that the dependence on the orientation is crucial, varying the orientation we change the order of thermalization time. This means that characteristic scale depends on the orientation. 

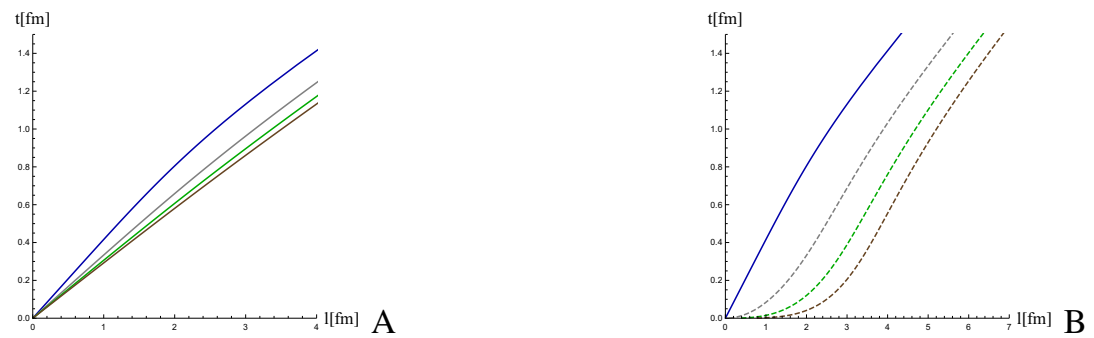

Figure 5. The thermalization time for the Wilson loop in the $x y_{1}$-plane with a short extent in the $x$ - and $y$ directions ((a) and (b), respectively). Different curves correspond to different values of $v=1,2,3,4$ (from top to down for each plot).
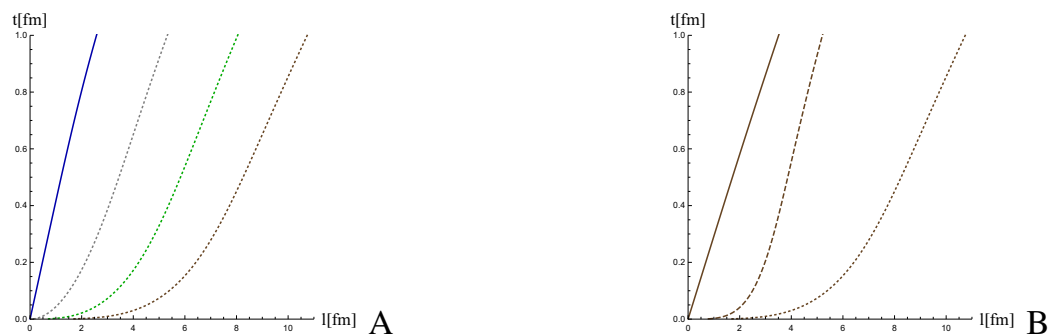

Figure 6. A. The thermalization time as a function of $\ell$ for the Wilson loop in the $y_{1} y_{2}$-plane, different curves correspond to different values of $v=1,2,3,4$ (from left to right). B. The thermalization time as a function of $\ell$ for $v=4$, for Wilson loops with a short extent in the $x$ - and $y$ - directions lying in the $x y_{1}$-plane and for Wilson loop in the $y_{1} y_{2}$-plane (from left to right).

\section{Conclusion}

As a conclusion let me summarize. Using conjecture that black hole production in AdS 5 gives quark gluon plasma formation in 4-dim QCD we have got:

- Multiplicity, that fits the recent experimental data [19, 20].

- The thermalization time on $\ell$ of two-point correlators as well as Wilson loops depends on orientation. It is not clear yet how we can check experimentally this prediction.

As to further directions of application of holography to quark gluon plasma formation in heavy ion collisions, I would like to mention the AdS/CFT approach to dealing with QGP formation with non-zero chemical potential [14]. In the context of the future project NICA this becomes really very actual.

\section{Acknowledgments}

I would like to thank the Organizers of Quarks2016 for the kind invitation and warm hospitality. This work is supported by the Russian Science Foundation (project 14-50-00005). 


\section{References}

[1] J. Casalderrey-Solana, H. Liu, D. Mateos, K. Rajagopal, U. A. Wiedemann, Gauge/String Duality, Hot QCD and Heavy Ion Collisions, Cambridge University Press 2014

[2] I. Ya. Aref'eva, Phys. Usp. 57 (2014) 527.

[3] O. DeWolfe, S. S. Gubser, C. Rosen and D. Teaney, Prog. Part. Nucl. Phys. 75, 86 (2014).

[4] A. Bazavov et al. Phys. Rev. D 90, 094503 (2014)

[5] J. M. Maldacena, Adv. Theor. Math. Phys. 2, 231-252 (1998) [hep-th/9711200].

[6] V. Matveev, R. Muradyan and A. Tavkhelidze, Lett. Nuovo Cim. 1972. V.5. P.712

[7] S. S. Gubser, S. S. Pufu and A. Yarom, Phys. Rev. D 78 (2008) 066014; JHEP 0911 (2009) 050

[8] D. Grumiller and P. Romatschke, JHEP 0808 (2008) 027 [arXiv:0803.3226 [hep-th]].

[9] L. Alvarez-Gaume et al., JHEP 0902 (2009) 009

[10] J. L. Albacete, Y. V. Kovchegov and A. Taliotis, JHEP 0807 (2008) 100

[11] S. Lin and E. Shuryak, Phys. Rev. D 79 (2009) 124015

[12] J. L. Albacete, Y. V. Kovchegov and A. Taliotis, JHEP 0905 (2009) 060

[13] I. Y. Aref'eva, A. A. Bagrov and E. A. Guseva, JHEP 0912, 009 (2009)

[14] I. Y. Aref'eva, A. A. Bagrov and L. V. Joukovskaya, JHEP 1003 , 002, (2010)

[15] Y. V. Kovchegov and S. Lin, JHEP 1003 (2010) 057;

Y. V. Kovchegov, Prog. Theor. Phys. Suppl. 187 (2011) 96

[16] E. Kiritsis and A. Taliotis, JHEP 1204, 065 (2012)

[17] P. M. Chesler and L. G. Yaffe, Phys. Rev. Lett. 106 (2011) 021601

[18] W. van der Schee, "Gravitational collisions and the quark-gluon plasma," arXiv:1407.1849.

[19] G. Aad et al. [ATLAS Collaboration], Phys. Lett. B 710, 363 (2012)

[20] J. Adam et al. [ALICE Collaboration], Phys. Rev. Lett. 116, no. 22, 222302 (2016)

[21] L.D. Landau, Izvestya AN. Ser. phys. 1751 (1953)

[22] Fermi E Progr. Theoret. Phys. 5570 (1950)

[23] I.Ya. Pomeranchuk, DAN, SSSR 788841951

[24] E.L. Feinberg, Phys. Usp. 168 (1998) 697.

[25] U. Gursoy, E. Kiritsis, L. Mazzanti and F. Nitti, JHEP 05, 033 (2009);

[26] I. Ya. Aref'eva, E. O. Pozdeeva and T. O. Pozdeeva, Teor. Mat. Fiz. 180, 35 (2014).

[27] D. S. Ageev and I. Ya. Aref'eva, J. Exp. Theor. Phys. 120, no. 3, 436 (2015)

[28] M. Strickland, Thermalization and isotropization in heavy-ion collisions, Pramana 84, (2015) 671.

[29] D. Giataganas, Observables in Strongly Coupled Anisotropic Theories, PoS Corfu 2012, 122 (2013).

[30] I. Ya. Aref'eva and A. A. Golubtsova, JHEP 1504 (2015) (011).

[31] I. Ya. Aref'eva, Theor. Math. Phys., 184:3 (2015), 1239-1255, arXiv:1503.02185.

[32] I. Y. Aref'eva, A. A. Golubtsova and E. Gourgoulhon, Analytic black branes in Lifshitz-like backgrounds and thermalization, arXiv: 1601.06046

[33] D. S. Ageev, I. Y. Aref'eva, A. A. Golubtsova and E. Gourgoulhon, "Holographic Wilson loops in Lifshitz-like backgrounds," arXiv:1606.03995.

[34] M. Taylor, Non-relativistic holography, arXiv:0812.0530.

[35] T. Azeyanagi, W. Li and T. Takayanagi, JHEP 06 (2009) 084.

[36] A. Donos, J. P. Gauntlett and C. Pantelidou, JHEP 1201 (2012) 061 